\title{
The Reliability of Plasma Exosome Concentrations in Healthy Male Individuals
}

\author{
Eman Abu-Seer \\ Oxford Brookes University, Gipsy Lane, Oxford, OX3 OBP, UK
}

\begin{abstract}
Exosomes are nanometer sized vesicles $(30-100 \mathrm{~nm})$, released from cells following the fusion of multivesicular bodies with the plasma membrane. Exosomes have a major role in intracellular communication, immune response modulation, physiological and pathological functions, and an important use in different drug therapies. Moreover, they have been identified as potential biomarkers and are involved in cancer and many other disease processes. Despite the developments in this area, no studies exist that assess the variability of plasma exosomes measures within and between subjects. The purpose of this study was to assess within and between day variability of plasma exosomes concentration in healthy individuals. Also, the stability of exosomes during freeze/thawing cycles was measured in this study. Eleven healthy men were assessed for reliability of exosome concentrations taken over the same sampling period, over 10 hours of a day and over five days. Exosomes were isolated by differential ultracentrifugation and characterized by their size distribution and morphology by electron microscopy and using the Nanosight tracking analysis respectively. Mean plasma exosome values were 1.505-2.245 $\left.\times 10^{\wedge} / \mathrm{mL}\right)$. Within day variability was not significantly different $(P=$ $0.95)$, and between day variability was not significantly different $(P=0.42)$. The interclass correlation coefficients (ICC) of 0.80 for within sample showed good reliability; ICC of 0.84 for between day plasma exosomes concentrations showed good reliability, and the within day ICC of 0.70 showed a moderate reliability. There was no significant difference observed in using fresh or frozen plasma in exosome quantification or exosome concentration. The statistics show that there is little significant variability in plasma exosome concentrations taken over a single sampling period, over a day or between days, which is vital for plasma exosome studies.
\end{abstract}

Key words:

\section{Introduction}

\subsection{Exosomes}

In normal cell's physiology, there is constant transport of biological materials across the cellular membranes. This transport works according to an evolutionary conserved mechanism and involves active and passive modes of transport along with the trafficking through micro particles, vesicles produced by the cell, including exosomes [1]. Exosomes are under the scientific spotlight as important methods of extracellular excretions and are nanometer sized vesicles (30-100 nm), released by cells, both normal and cancerous, into the extracellular space by inward budding of endosomal membrane into structures called multi-vesicular bodies (MVBs), followed by fusing of

Corresponding author: Eman Abu-Seer, research field: clinical trials.
MVBs with the plasma membrane and release their internal component (exosomes) extracellularly [2]. They are composed of nucleic acid and protein and have been largely attributed to be the carriers of these constituents to various parts of the body without any discrimination. However they are also widely known to carry intercellular messages, effects of and signalling macromolecules between highly specific set of cells [2]. Exosomes were first reported by Johnstone and his colleagues in 1987, when they found that exosomes are secreted from reticulocytes during maturation [3]. Exosomes can be released by many cells, such as T-cells, dendritic cells, and oligodendrocytes cells and non-hematopoietic cells such as neuroglial cells, tumour cells and intestinal epithelial cells [4]. They have been detected in several biological fluids as plasma, saliva, nasal lavage, placenta, and in breast milk [5]. Exosomes levels in plasma are clinically 
interesting in many applications for example the plasma levels in some cancer patient could be a potential tool for cancer screening [6].

\subsection{Exosomes as Potential Biomarkers in Cancer Identification and Development}

It has been largely hypothesized that since the exosomes carry a particular cellular content, they can be exploited as potential biomedical tools to detect the changes in the content of the cells, as in the initiation and progression of cancer or tumorous growths. The micro ribonucleic acid content of the exosomes corresponds to only particular cell types and are attributed to changing the phenotype of the progenitor cells by effectively transferring pro-oncogenic molecules in order to initiate the growth of cancers in these cells and hence give rise to metastatic environment in the neighbouring cells [7]. It has been suggested that increased level of exosomes can be detected in urine, serum, and in malignant effusions from cancer patients [8]. Kharaziha, Ceder [9] reported that cancer cells secrets higher amounts of exosomes than normal cells. Furthermore, Taylor and Gercel-Taylor [10]found that cancer patients have excessive amounts of blood circulating exosomes compared to healthy individuals. Therefore, the number of exosomes is important and can be used as markers in many diseases including cancer. Logozzi, De Milito [6] study shows high levels of exosomes that expressed CD63, housekeeping protein present in all human beings nonetheless, and Caveolin-1, tumour-associated biomarker, in the plasma of cancer patients. ELISA was used for quantifying the levels of exosomoes present in the plasma of the cancer patients that expressed the two proteins and it was found that the levels for CD63 and Caveolin-1 were significantly increased in melanoma patients as compared to the healthy individuals who donated plasma. Therefore, the analysis of exosomes for specific tumour-biomarkers can help in the early detection of cancer before it develops into a full-grown metastasized tumour and causes mortality [6]. Furthermore, Ren and colleagues were able to show that exosomes present in human plasma displayed a variety of shapes and exhibit the exosome marker protein $\mathrm{CD} 81$ besides $\mathrm{CD} 63$, along with other members of MHC Class II including CD86, Wnt3a, Wnt5a and FasL which are normally known as the cell signal transduction molecules [11]. Plasma exosomes were also shown to participate in the inhibition of CD4+ $\mathrm{T}$ cells involved in the immune system. They possess the ability to induce suppression, in dose-dependent manner, of the active CD4 $+\mathrm{T}$ cells and their proliferation in the plasma, leading to their natural apoptosis in the body [11]. Moreover, Salma Khan and colleagues researched on the levels of Exosomal Survivin in patients with prostate cancer and in control patients, derived from their plasma. Survivin is expressed in the plasma of patients diagnosed with prostate cancer and it is important for making the $\mathrm{PCa}$ cells sensitive to the chemotherapeutic agents. Its downregulation causes the effective eradication of the cancerous cells at the end of chemotherapy. The recent research in the exosomes has shown that the exosomes derived from the tumour, much like the ones derived in this experiment, help drastically in the initiation and development of cancer in the cells affect as well as help it metastasize to other parts of the body. Exosomes were purified from serum and plasma samples from PC patients as well control population using the experimental techniques of ultracentrifugation and ELISA and Western blotting were used to quantify the amount of exosomes present in the samples isolated. It was found to be present in elevated levels in the tumour-derived samples as compared to the serum and plasma exosome levels in the control patients. The patients who had failed to respond to chemotherapies also projected increased levels for exosome-derived Survivin. Therefore, the researchers were successful in showing that Survivin exists in the plasma and serum-derived exosomes in 
elevated amounts and can be used as a potential biomarker in early detection of cancer [12].

\subsection{Additional Functions of Exosomes}

In addition to the interest in exosomes as potential biomarkers, their physiological roles are also important which basically deal with the pathways related to intracellular and intercellular communications. They play a crucial role in cell to cell communication when they interact with other cells by carrying proteins, lipids, and genetic information [13], whether the cells present locally or at a distance. They have been found to be involved in immune regulation and act as antigen-presenting vesicles, the behaviour commonly found in the $\mathrm{B}$ and $\mathrm{T}$ immunity cells, in order to stimulate the immune response and induce tolerogenic effects [13-15]. Also exosomes have been involved in some diseases' pathogenesis such as neurodegenerative disease and cardiovascular disease [16]. It has been shown that exosomes are involved in the accumulation of $\beta$-amyloid protein which is associated with Alzheimer's' disease [8].

Recently a wide range of exosomes physiological and pathological function studies have been conducted. Although the number of studies examining plasma exosomes is increasing, however, there is a need to better understand the variables that affect exosomes levels and the mechanisms that control the production and mediation of exosome levels. Also there might be variation in between subjects over a day and within a day. Although, circadian variations are often detected on human immune biomarkers and have been studied. For example, the study conducted by Ündar, Ertugùrul [17] reported a significant circadian variation in different blood biomarkers (IL-6, coagulation inhibitors proteins, and anti-thrombin factors in healthy men [17]. However, exosomes' circadian rhythm in healthy individuals is still unknown.

It is important to know the variability in measuring exosome numbers and size. The variability can help point towards the variety of roles that exosomes perform in the various parts of the human body in their multiple morphological orientations as demmed appropriate and essential based on the biochemical environment inside and outside the cells. Further which measurement bias in the data affects the measurement variability, which can occur in pre-analytical (specimen collection, handling and processing), analytical (imprecision and bias), and post-analytical (interpreting and reporting data) data sets [18]. Gardiner, Ferreira [19] examined the analytical measurement variation of exosomes measures processes by Nano sight Tracking Analysis (NTA). It is essentially a technique where the light is scattered by the subjects in focus which leads to the specification of their size and quantity present in a sample. They used silica microspheres in order to calibrate the measurements taken by the technique as standardized method for extra-vesicles (exosomes) measurements, in order to decrease the level of inaccuracy associated with the measurements. Gardiner, Ferreira [19] showed data from several repeat measurements of known concentration. However, a biological standard for exosomes measurement has not been studied and the within-subject variations have not been addressed. The additive features of the technology are distributed across five distinct levels. Primarily, it allows for determining the detection threshold which refers to the minimum value for the intensity of an image that can help distinguishing a particle for further analysis. Furthermore, the technology can help in determining the maximum distance that the software expects a particle to move from one frame of reference to another. This is directly proportional to the size of the particle and therefore, the larger particles would take longer to move across the frames. This minimum expected particle size is automatically determined by the software. Moving on, blur which defines the degree of smoothing of the images received by the software after removing the unrequired noise, including predominantly the diffraction rings found around the 
larger particles, is another automatic setting built into the software. It can be manually adjusted to increase for bigger particles and decrease for the smaller ones. Moreover, it also determines the minimum track length that a particle should cover based on its size for it to be included in the size distribution plot. This plot is entirely based on the Mean Square Displacement (MSD) profile for all the particles and which have been tracked for a minimum number of frames as determined by the machine automatically or set manually by the scientific researcher. Higher values for minimum track length mean that the particles have been tracked for a greater length of time which helps in accurate sizing of all the particles under scrutiny. However, it does not imply that the particles that spend less time in crossing one frame of reference to another are measured inaccurately. It can also mean that these particles are smaller in size and hence, take less time to get across the threshold. Lastly, the background noise or pixel contamination can be easily removed from the extracted data sets or images in order to make the readings as accurate as possible for the size of the particles. Therefore, Nanoparticle Tracking Analysis can be successfully employed to determine size of the biological molecules such as exosomes to develop the importance of their molecular physiology to the function they perform [19].

Considering physiological and pathological importance of exosomes as potential biomarkers for neurodegenerative diseases and multiple cancer types, there exists a further need for reliability measures of plasma exosomes concentration in plasma, thereby leading to reliable and accurate prognosis of disease initiation and development. In conjunction to their need, the day-to-day variation in the readings should also be well accounted for and measured accurately to understand their association with changing biochemical environment in the body and how the exosomes can help as effective tools in marking these changes at early stages. This will help to determine whether a single fasted blood sample is sufficient or if consecutive day blood samples are needed to present an individual's exosomes status. Therefore, the purpose of this study is to assess within and between day reliability of plasma exosome concentration. We hypothesized that plasma exosomes concentration should be stable within sample, although there might be little within day variability, however the overall plasma exosome concentration is found to be stable over the period of five days of blood sampling in a week.

\subsection{Aims and Objectives of the Study}

The main aim of this study is to explore reliability of plasma exosomes in healthy individuals. There are other objectives of the study:

- To assess within sample, within day and between days variability of plasma exosome concentrations

- To predict the size distribution for a given population of extracellular vesicles within a day at different time points and also between days over five consecutive days in healthy individuals.

- To determine the stability of exosomes during freeze/fresh plasma.

Although, circadian variations are often detected on human immune biomarkers. We hypothesized that plasma exosomes concentration should be stable within sample; there would be little day to day variability in plasma exosomes concentration over the five days of blood sampling.

\section{Methods}

\subsection{Participants}

Eleven healthy male participants (age $27.6 \pm 4.2 \mathrm{y}$, height, $177.0 \pm 9.4 \mathrm{~cm}$, and body weight, $80.3 \pm 12 \mathrm{~kg}$ ) volunteered to participate in the study which was approved by the University Research Ethics Committee. This study conformed with the latest revision of the Declaration of Helsinki. All procedures and potential risks associated with the study were fully explained to each participant. All participants reported to the human performance laboratory and answered 
screening questionnaires to determine their eligibility for the participation in the study. Participants were eligible for inclusion if they were healthy male and between 20 and 60 years of age. Exclusion criteria included diagnosis of a chronic inflammatory condition (e.g diabetes mellitus and cardiovascular diseases or recent consumption of anti-inflammatory medications (e.g ibuprofen). Since the scope of the study is to study the variation found in the levels of exosomes or their concentration in healthy individuals, therefore the indication of any inflammatory condition refers to the natural increase in the concentration of exosomes and any proteins that are associated with anti-inflammatory response [20]. Moreover, subjects were excluded if they had any pathology known to influence immune response (e.g. Auto immune diseases, anaemia, heart diseases, orthopaedic limitations or renal failure disease), or neurological, neuromuscular disorder. Subjects who had any cold or flu symptoms in the previous $24 \mathrm{~h}$ were excluded from the study based on the same explanation as provided earlier about the natural increase in the level of exosomes in cases of inflammatory response.

\subsubsection{Sample Size}

This is part of another study (exosome and inflammatory markers (TNF-a, IL-6, IL-10) in healthy individuals before and after high/low intensity exercise), in which the same sample size calculation was also used in this study. The sample size calculation is based on data from a review which has been done by [21], based on cytokines (TNF-a, IL-6, IFN- $\gamma$ ) measurement between healthy and healthy group. With an effect size of 0.55 , the power of this study is set at $80 \%$, and the level of significance is set at $5 \%$ (0.05, two tailed-analyses). Also we used sample size of 11 individuals based on a previous study by [22], which looked at changes in muscle RNAs in response to acute and chronic aerobic exercise.

\subsubsection{Within Sample}

For all studies, during each visit individuals were asked to fast for 8 to 10 hours before blood draws and refrain from alcohol for $24 \mathrm{~h}$, and an exhaustive exercise for $48 \mathrm{~h}$ before each visit. They were allowed to drink water only. In order to determine whether the method of extracting exosomes were reproducible during each visit, three blood samples ( $4 \mathrm{~mL}$ each) were drawn consecutively from the antecubital vein into EDTA vacutainers (Becton Dickinson; San Jose, California). Then blood sample was centrifuged at $1,000 \mathrm{~g}$ for $10 \mathrm{~min}$ at $4{ }^{\circ} \mathrm{C}$, plasma was separated, divided into $500 \mu$ aliquots and subsequently stored at $-80{ }^{\circ} \mathrm{C}$. The exosomes were then processes as below.

\subsubsection{Within Day Reliability}

In order to assess within day reliability, the same eleven individuals were participated in this study. In this visit, the first fasted blood sample was drawn by venepuncture using a winged infusion device at the baseline $0 \mathrm{~h}$. Then blood sample $(4 \mathrm{~mL})$ was drawn at 1 h, 2 h, 3 h, 4 h, 5 h, 6 h, 8 h, and at 10 h. Participants were offered a standardized diet after the second hour and the sixth hour from the beginning of the study. All blood samples were collected, and processed as stated above.

\subsubsection{Between Day Reliability}

In order to examine plasma exosomes reliability between multiple days, same eleven participants reported to the laboratory five times over five consecutive days. During each visit blood sample (4 $\mathrm{mL}$ ) was drawn at the same time from every participant. All blood sample were processed same as stated above.

\subsection{Study Design}

\subsubsection{Preparation of Plasma Exosomes}

Exosomes were isolated by differential ultracentrifugation. Basic exosomes isolation protocol was followed as described by [23]. Plasma sample was separated from blood and diluted with an equal amount of PBS and gently mixed until homogenous. Then the mixture was transferred in $50-\mathrm{mL}$ tubes, centrifuged for $30 \mathrm{~min}$ at $2,000 \times \mathrm{g}, 4{ }^{\circ} \mathrm{C}$ followed by at $12,000 \mathrm{~g}$ for $45 \mathrm{~min}$. The suspension was filtered 
through a $1 \%$ BSA blocked $0.22-\mu \mathrm{m}$ filter, collected in a fresh ultracentrifuge tube, and centrifuged for $2 \mathrm{~h}$ at $110,000 \times \mathrm{g}, 4^{\circ} \mathrm{C}$ in a Beckman LE-80K centrifuge, swing out rotor (Beckman Coulter Optima, SW 32 Ti). Subsequently the pellet was washed in PBS by filling up a $14 \mathrm{~mL}$ tube with PBS and then centrifuged at $110,000 \times \mathrm{g}, 4{ }^{\circ} \mathrm{C}$ for $70 \mathrm{~min}$. After, the pellets were re-suspended in $50 \mu \mathrm{PBS}$, and stored at $-80^{\circ} \mathrm{C}$.

Exosomes were immediately isolated from the first aliquted samples (A) by differential ultracentrifugation and then examined morphologically by electron microscopy. The number of exosomes were analysed by Nanosight tracking analysis. The second plasma samples (B) were

\subsection{Exosomes Analysis}

\subsubsection{Stability of Plasma Exosomes}

To determine the stability of exosomes during freeze/fresh plasma $(5 \mathrm{~mL})$ from eleven healthy individuals were collected and divided into two $2.5 \mathrm{~mL}$ samples. Then exosomes were immediately isolated from the first aliquted samples (A) by differential ultracentrifugation and then examined morphologically by electron microscopy. The number of exosomes were analysed by Nanosight tracking analysis. The second plasma samples (B) were aliquted and stored at $-80^{\circ} \mathrm{C}$ for three months. Exosomes were isolated, morphologically tested and analysed by NTA as prescribed below (preparation of plasma exosomes, and NTA).

2.3.2 Preparation of Plasma Exosomes (Isolation and Extraction)

Exosomes were isolated by differential ultracentrifugation. Basic exosome isolation protocols were followed as described [24]. A plasma sample was separated from blood by spinning whole blood at 1,000 $\mathrm{g}$ for $10 \mathrm{~min}$ in Biofuge primo ( Heraeus instrument). Then plasma was diluted with an equal amount of PBS (Sigma 764) and gently mixed until homogenous. Then the mixture was transferred in 1,5 $\mathrm{mL}$ Eppendrof tubes, centrifuged for $30 \mathrm{~min}$ at $2,000 \times \mathrm{g}, 4{ }^{\circ} \mathrm{C}$ (Biofuge fresco, Heraeus instrument). Then centrifuged at $12,000 \mathrm{~g}$ for $45 \mathrm{~min}$ in a Beckman LE-80K centrifuge (Beckman Coulter Optima, swinging bucket rotors). The suspension was filtered through a $0.22-\mu \mathrm{m}$ filter (pre-block with $1 \% \mathrm{BSA}$ ), collected in a fresh ultracentrifuge tube, and centrifuged for $2 \mathrm{~h}$ at $110,000 \times \mathrm{g}, 4{ }^{\circ} \mathrm{C}$ in a Beckman LE-80K centrifuge (Beckman Coulter Optima, swinging bucket rotors). Subsequently the pellet was washed in PBS by filling the tube up to the top with PBS, and then centrifuged at $110,000 \times \mathrm{g}, 4^{\circ} \mathrm{C}$ for $70 \mathrm{~min}$ (Beckman Coulter Optima, swinging bucket rotors). After, the pellets were re-suspended in $50 \mu \mathrm{PBS}$, and store $\mathrm{d}$ at $-80^{\circ} \mathrm{C}$.

\subsubsection{ExoQuickTM Precipitation}

Exosome isolation from plasma ExoQuickTM precipitation was carried out according to manufacturer's instructions (System Biosciences). Briefly, $500 \mathrm{~mL}$ of clarified CCM was diluted to $5 \mathrm{~mL}$ in PBS and mixed with $1 \mathrm{~mL}$ of ExoQuick-TCTM solution by inverting the tube several times. The sample was incubated overnight at $4{ }^{\circ} \mathrm{C}$ then centrifuged twice at $1,500 \mathrm{~g}$ for 30 and $5 \mathrm{~min}$, respectively, in order to remove the supernatant. The supernatant was discarded, and the pellet was resuspended in $200 \mathrm{~mL}$ of PBS.

2.3.4 Transmission Electron Microscopy (TEM) of Exosomes Samples

An aliquot of exosomes vesicle sample was combined 1:1 with 4\% PFA (Sigma, 158127) and cooled for $15 \mathrm{~min}$ on ice. Then a single drop of each sample was placed onto a strip of Para film (VWR, 52858). Carbon-formvar coated copper grids, 200 mesh (F077, TAAB) were placed dull-side down onto the exosomes vesicles/PFA drop and left at room temperature for 30-45 min. Grids were then placed sample down onto three $30 \mu \mathrm{l}$ drops of $0.22 \mu \mathrm{m}$ filtered ultrapure water for one minute each. the side of the grids were gently touched to a filter paper between each drop to remove excess solution. Then grids were placed sample-side down onto a $30 \mu \mathrm{L}$ drop of $2 \%$ uranyl acetate (aq) for two minutes. All grids were 

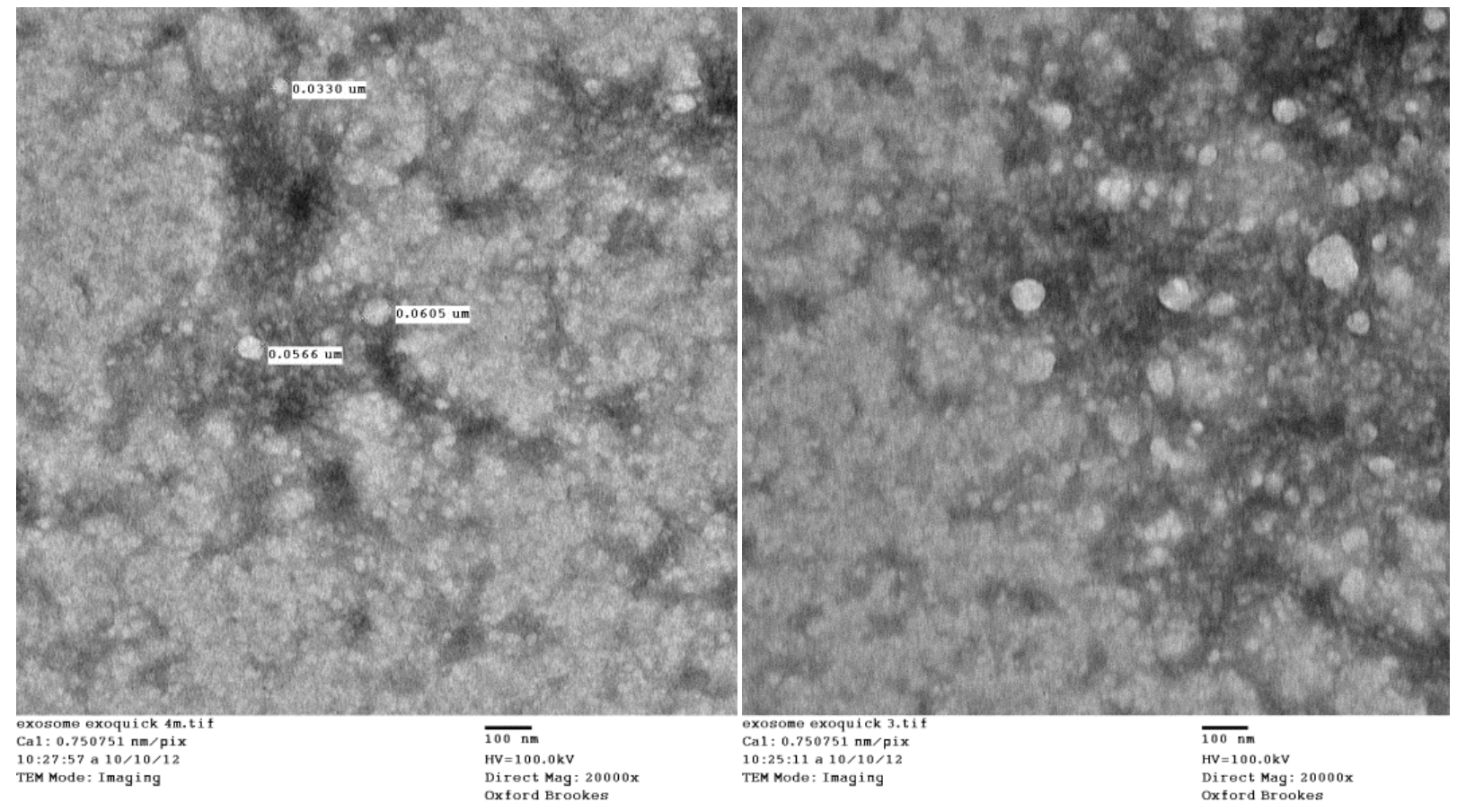

Fig. 1

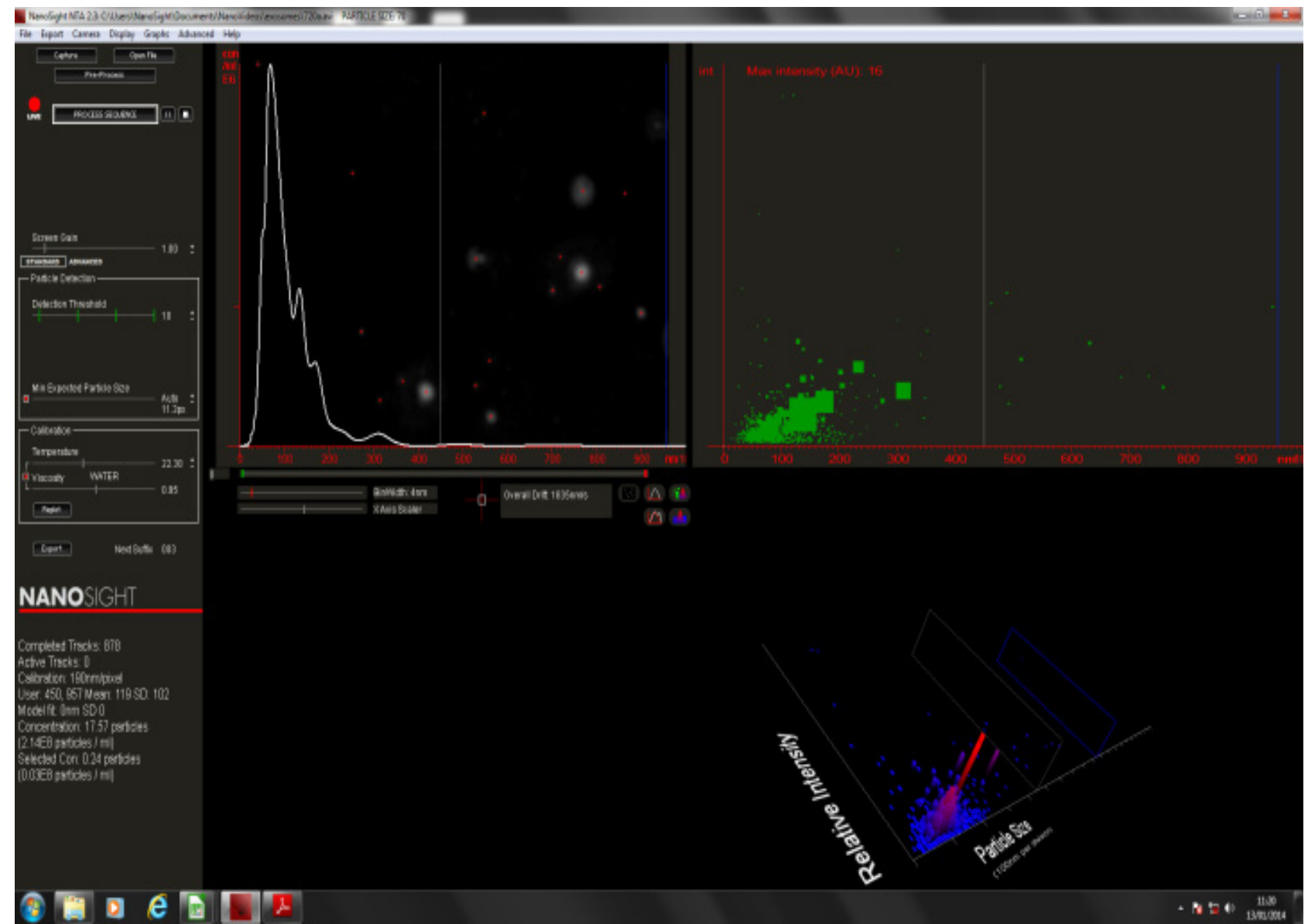

Fig. 2 NTA measurement of particle size and concentration. 


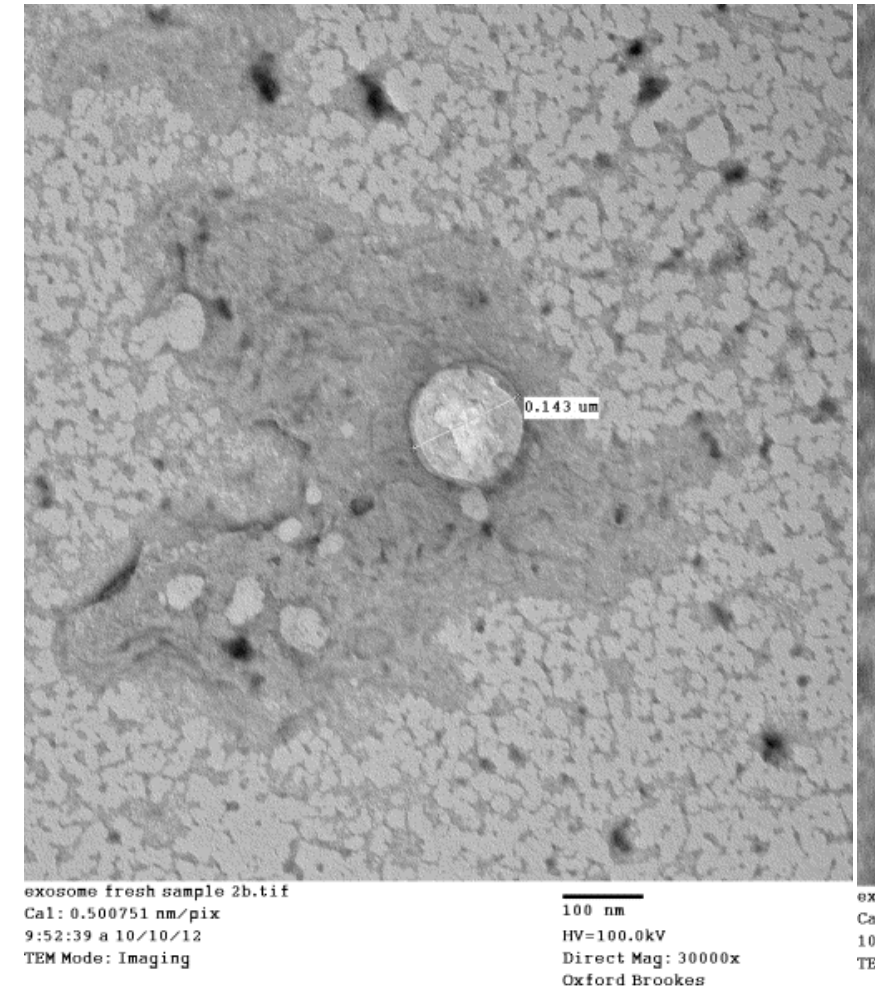

(a)

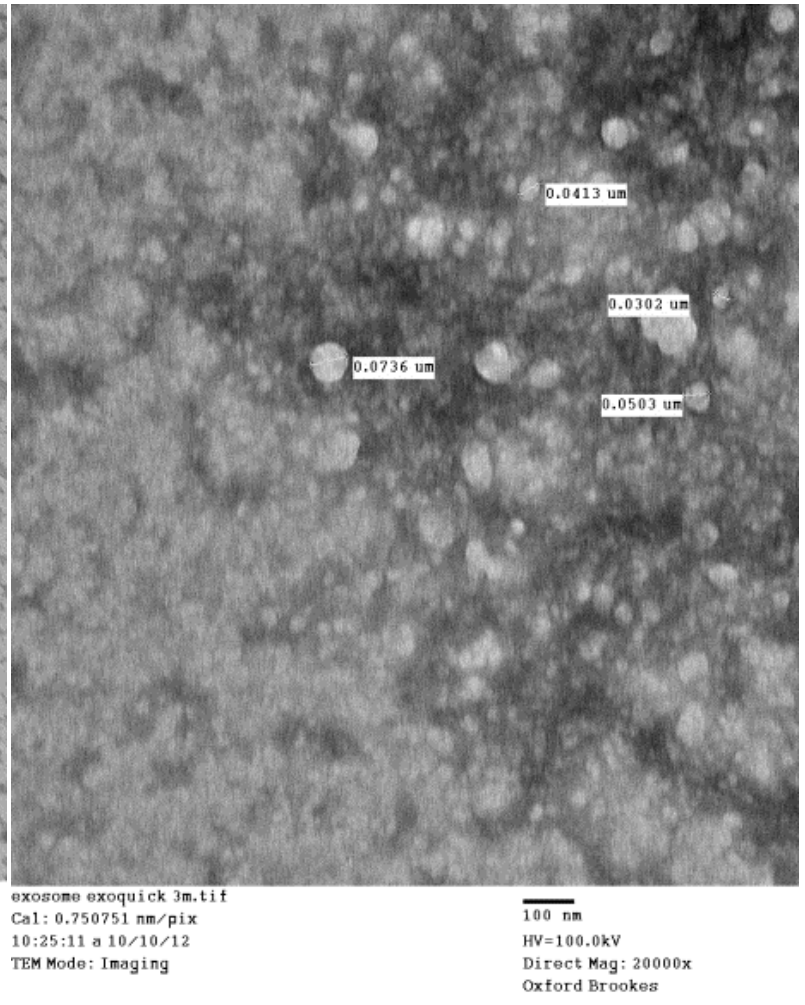

(b)

Fig. 3 A. fresh exosomes shown by electron microscopy, B. Frozen exosomes.

touched to the filter paper and left to dry, in which sample side up for one hour. After that, grids were stored in a box for analysis by using a Hitachi H7650 Transmission Electron Microscope (TEM) at $120 \mathrm{kV}$.

\subsubsection{Nanoparticle Tracking Analysis (NTA)}

Sample suspensions containing exosome vesicles were first diluted 1:500 PBS, kept on ice, then analysed using a Nanosight LM10, NS500 instrument (NanoSight, Amesbury, UK). For this, 90 second videos were used to track the Brownian movement using Version 2.2 Nanosight software to give mean and median vesicle size and concentration (Fig. 2). For every sample this reading was done five times with mixing in between and then an average taken to remove sample mixing bias.

\subsection{Statistical Analysis}

Participant's characteristics data are presented as mean \pm standard deviation (SD). Statistical analyses were completed in SPSS for windows version 21
(SPSS Inc., Chicago, IL, USA). All variables were examined with one-way repeated measures ANOVA with time as the independent variable to detect any differences within day (time points $0 \mathrm{~h}, 1 \mathrm{~h}, 2 \mathrm{~h}, 3 \mathrm{~h}, 4 \mathrm{~h}$, $5 \mathrm{~h}, 6 \mathrm{~h}, 8 \mathrm{~h}$ and $10 \mathrm{~h}$ ) or between days (day 1, 2, 3, 4 and 5). Within day (across all nine time points; $0 \mathrm{~h}, 1 \mathrm{~h}$, $2 \mathrm{~h}, 3 \mathrm{~h}, 4 \mathrm{~h}, 5 \mathrm{~h}, 6 \mathrm{~h}, 8 \mathrm{~h}$ and $10 \mathrm{~h}$ ) and between day (across all five visits) reliability were examined with a two-way mixed effect models interclass correlation coefficient $\left(\mathrm{ICC}_{3,1}\right)$ for absolute agreement [25]. The ICC $95 \%$ confidence intervals $(95 \% \mathrm{CI})$ were also calculated. An ICC $\geq 0.7$ was accepted as moderate reliable as $0.6-0.8$ considered as moderate reliability and $\geq 0.8$ as good reliability. Coefficient of variation (CV) was determined for each participant using the formula: $\mathrm{SD} /$ mean $\times 100$ to determine measurement variability. Within day CVs were calculated across the time points. Within sample and between day CVs were calculated across the five visits and with the group average presented. Significance was set at the level of $P<0.05$. 


\section{Results}

\subsection{Stability of Plasma Exosomes}

In order to assess the stability of plasma exosomes under different conditions, fresh and frozen plasma exosomes were isolated by differential ultracentrifugation as described above. Then they were analysed by NTA showed a particle size ranging from 30 to $300 \mathrm{~nm}$ in diameter with an average of 120 $\pm 70 \mathrm{~nm}$. The number of fresh and frozen plasma exosome was stable (fresh samples $3.68 \pm 3.1$, frozen samples $4.36 \pm 2.8)$, there was no significant change between fresh and frozen plasma exosomes $(P=0.072)$ in all eleven samples which also analysed by NTA (Fig. 2). Under the electron microscopy, exosomes were appeared as spherical vesicles with a cup shape, also their diameters ranging from 30 to $120 \mathrm{~nm}$ (Fig. 3).

Reliability of plasma exosomes measures within sample, within day, and between days

This study was conducted to assess within and between day reliability of plasma exosomes concentration in healthy individuals. Eleven healthy men were assessed for reliability of exosome concentrations taken over the same sampling period, over 10 hours of a day and over five days. Table 1 shows the descriptive characteristics of all the eleven subjects who completed the study. No one had reported of any illness in the $24 \mathrm{~h}$ prior to the blood draw. All subjects were non-smokers.

Reliability and variability of plasma exosome concentration within the same subject at a single time point

Within sample variability of blood plasma exosomes concentration in three different samples withdrawn consecutively from each subject was measured using the nanosight.

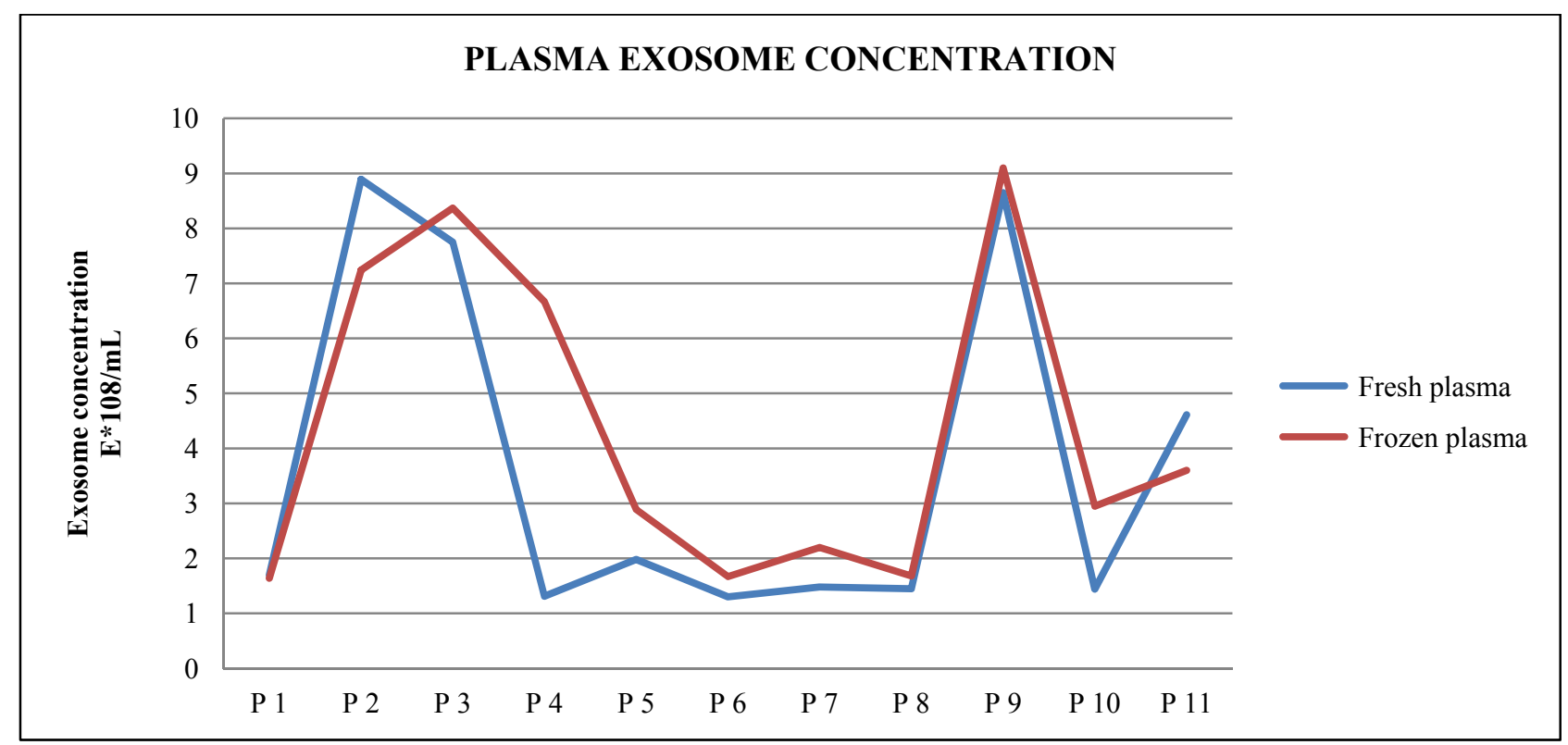

Fig 2 shows the number of exosomes which were measured under different conditions (fresh and frozen sample).

Table 1 Subjects characteristics.

\begin{tabular}{ll}
\hline Number of subjects & 11 \\
\hline Age (years) & $27.6 \pm 4.2$ \\
Height $(\mathrm{cm})$ & $177.2 \pm 9.4$ \\
Weight $(\mathrm{kg})$ & $80.3 \pm 12$ \\
Blood pressure (mm Hg) & $130 / 73 \pm 7.37$ \\
Heart rate (beat $/ \mathrm{min})$ & $90 \pm 30$ \\
\hline
\end{tabular}

*Data is reported as mean \pm standard deviation (SD). 
Table 2 Within sample, between and within day measures of plasma exosomes concentration.

\begin{tabular}{lllll}
\hline & $\begin{array}{l}\text { ANOVA } \\
\text { PVALUE }\end{array}$ & ICC & $\begin{array}{l}\text { 95\% CONFIDENCE } \\
\text { INTERVALS (CI) }\end{array}$ & $\begin{array}{l}\text { Coefficient of variation } \\
(\mathrm{CV}) \%\end{array}$ \\
\hline Within Sample & 0.67 & 0.88 & $0.24-0.9$ & 42 \\
Between Day & 0.42 & 0.84 & $0.827-0.926$ & 34 \\
Within Day & 0.95 & 0.75 & $0.457-0.924$ & 30 \\
\hline
\end{tabular}

CV showed as SD/Mean $\times 100$.

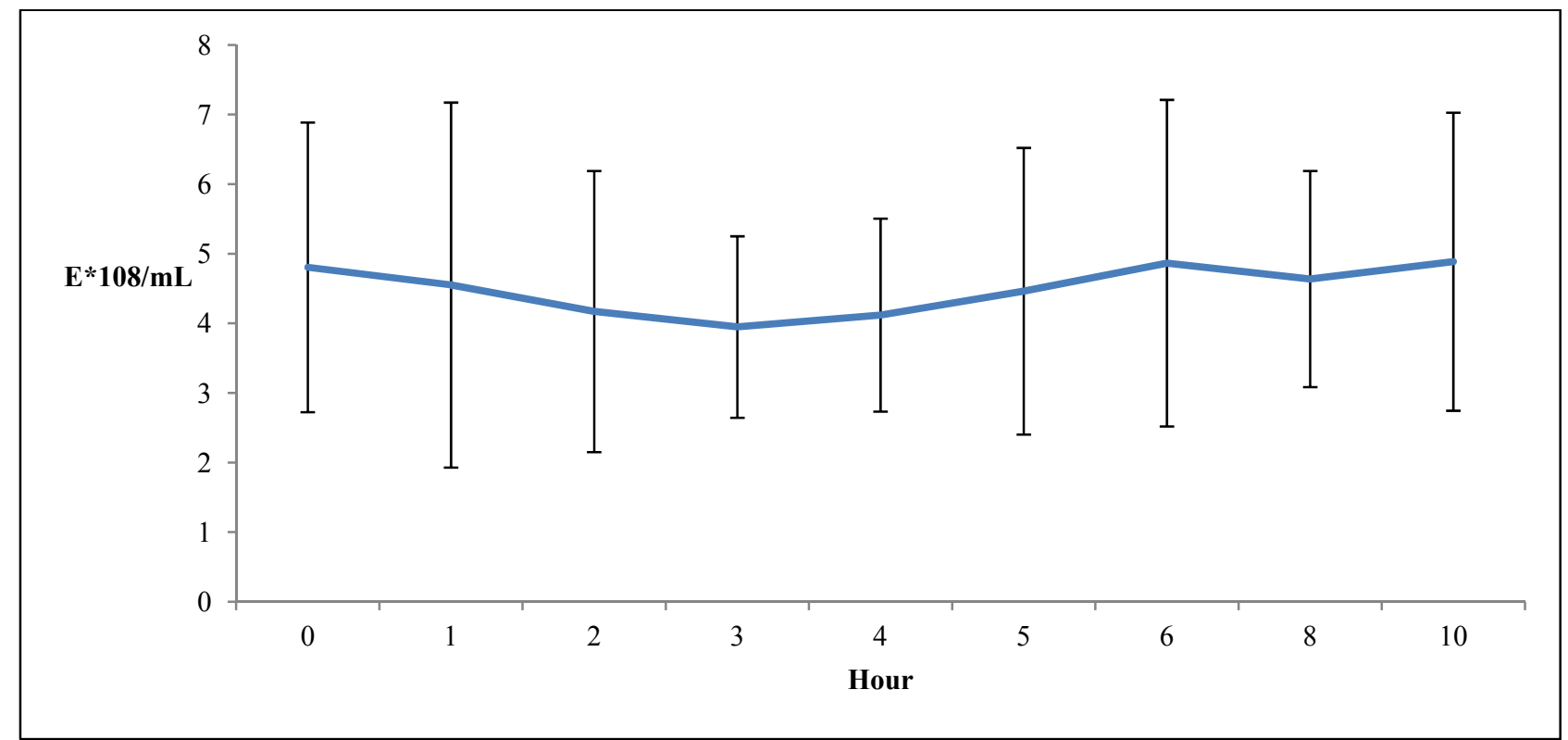

Fig. 2 Plasma exosome concentration of nine samples within one day over many hours showing the average of eleven participant samples, each replicate was measured five times on the Nanosight ( represented as mean \pm standard deviation).

The results from single level (sample-variation) of a repeated measures ANOVA test was performed in this study show that there was no significant difference between the three different repetitions of blood collection $(P=0.676)$. The reliability of plasma exosomes measures showed good within samples reliability (ICCs $\geq 0.88$ ) and minimal variability within sample (Coefficient of variation $\mathrm{CV}=42$ ) (Table 2). Therefore, our results show good and reliable results in plasma exosomes concentration within consecutive samples.

Reliability and variability of plasma exosome concentration and size within the same subject at multiple time points of the day

To assess within day reliability of exosomes concentration we measured plasma vesicles over 10 hours from each subject. The size distribution for exosomes was also measured within a day at different time points and also between days over five consecutive days in healthy individuals.

A single level repeated measures ANOVA test was performed in this study. The results show that there was no significant differences at any time point $(P=$ $0.95)$. The average exosomes values were $2.24 \times$ $10^{\wedge} / \mathrm{mL}$ for all time points in all subjects (represented in Fig. 2). Moreover, the reliability of within day plasma exosomes measures showed a moderate reliability ( $\mathrm{ICCs} \geq 0.75, \mathrm{CV}=34 \%$ ). For size distribution, the results shows that there was no change in the average size distribution at any time point of the day $(P=0.014)$.

Reliability and variability of plasma exosome concentration and size over multiple days

The reliability and variability of plasma exosomes measures can be seen in Table 2, demonstrated good reliable measure of plasma exosome (ICCs $\geq 0.84$ ) 
Table 2 Exosome concentration represented as mean \pm standard deviation of averages of eleven subjects per each day over five consecutive days.

\begin{tabular}{lll}
\hline & Mean \pm SD & $P$ value \\
\hline Number of subjects & 11 & \\
Average D1 & $1.52 \pm 0.74$ & $P=0.64$ \\
Average D2 & $1.58 \pm 0.96$ & $P=0.63$ \\
Average D3 & $2.53 \pm 2.37$ & $P=0.67$ \\
Average D4 & $1.42 \pm 0.84$ & $P=0.57$ \\
Average D5 & $1.94 \pm 1.88$ & $P=0.50$ \\
\hline
\end{tabular}

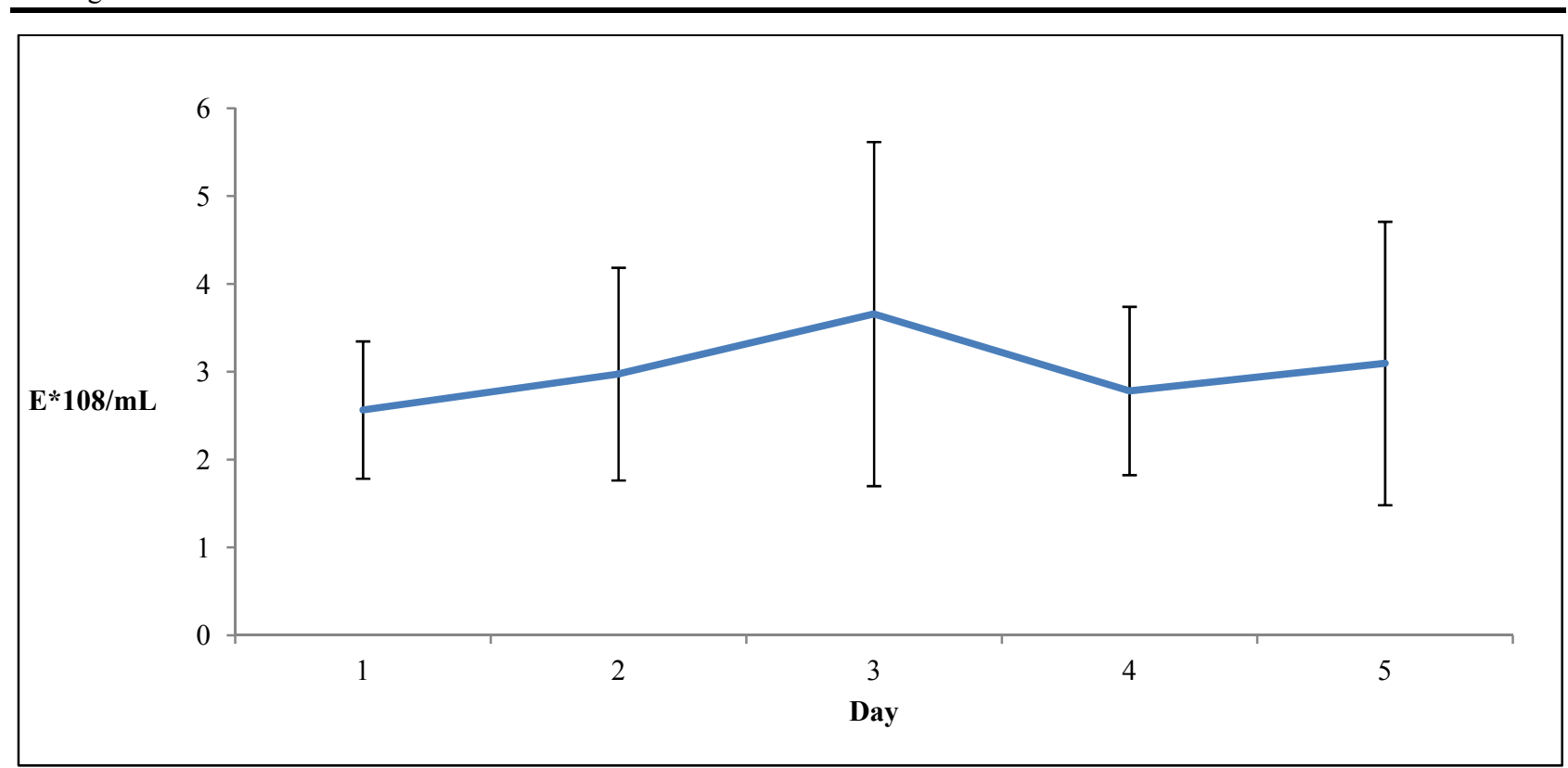

Fig. 3 Plasma exosome concentrations taken from the average of eleven participant samples over five consecutive days, each replicate was measured five times on the Nanosight (represented as mean \pm standard deviation).

And $\mathrm{CV}=30$ ). Over five days the average exosomes values of each sample were $1.505 \times 10^{\wedge} / \mathrm{mL}$. There was no significant difference in between day concentrations $(P=0.423)$. One-way analysis of variance (ANOVA) of the exosomes data also showed no significant effects differences between days (represented by the average value for each of the five days) is represented in Fig. 3. Moreover, there was no change in the average size distribution of all eleven subjects over the five consecutive days. These data represented in Table 2 .

\section{Discussion}

In this study, we examined the within and between subject reliability of plasma exosome concentrations. As stated by Hopkins [26], that reliability refers to the reproducibility of values of a measurement in repeated trials on the same subject. However, Batterham and George [27] state that within subject variation, systemic change in the mean, and re-test correlations are the main measures of reliability. First, we examined the within sample reliability in three different samples withdrawn at the same time from each individual. There was no significant difference between the three different repetitions of blood collection $(P>0.05)$, which suggest that there is no significant variability explained by technical issues with blood sampling as it showed good reliability. Second, we examined the within day reliability through consequent measures of plasma exosomes over different time points (10 $\mathrm{h}$ monitoring). We found that there was no significant difference at any 
time point, which suggests that exosomes are considerably stable within the day, also there was no significant differences between day measures. We found that average plasma exosomes concentration was ranged between 1.505-2.245 (vesicles $\times 10^{\wedge} / \mathrm{mL}$ ).

Our results are demonstrated that measurement of plasma exosomes concentration is reliable and repeatable in healthy subjects. The ICCs were $\geq 0.8$ for within sample and between day measures, which shows good reliable measures of plasma exosomes concentration. Whereas ICCs of $\geq 0.75$ was moderate for within day reliability. We also examined the stability of plasma exosomes under different conditions. Our results showed that freeze condition had no effect on size and number of plasma exosome. These results are consistent with Kalra, Adda [28], they assessed the stability of exosomes in plasma under various storage conditions and over 90 days. In which fresh and frozen plasma samples were obtained from five healthy donors and then blood plasma was pooled and prepared for exosomes isolation. Furthermore, a study conducted by [29] also tested the stability of exosomes during freeze thaw cycles, however plasma samples were collected from healthy pregnant women. Further to exosomes isolation by differential and sucrose density gradient centrifugation, they also measured common proteins identified in exosomes isolated from fresh plasma and after freeze/thaw cycles [29]. Exosomes are stable after a freeze and thaw cycle in healthy women during pregnancy. Recently, several studies investigated the stability of exosomes and also circulating miRNA from exsomes in plasma. It is therefore indicate that exosomes can be good biomarkers according to their stability under various conditions [29]. Finally we analysed plasma exosomes based on particle size. Data were divided into two categories, vesicles size ranges between $30-100 \mathrm{~nm}$ terms as exosomes and microvescicles for vesicles size ranged between $101-1,000 \mathrm{~nm}$. There was no change in the number of both extracellular vesicles. Also there was no significant change in exosomes size within the same subject at multiple time points of the day and over multiple days.

There were several limitations which may contribute to increased variability in the measurement of plasma exosomes. First, there was no dietary control and dietary record for what they had in the day before. However, we attempted to control this by asking all the participants to be in a fasted state and by collecting the blood samples at the same time of each day over the five consecutive days. Second, the variability may be due to the number of physiological fluctuations which associated with an impairment of some immune parameter's response [30]. Therefore, stress is one of the physiological factors that fluctuate from day to day which could be a possible cause for the variability in exosomes concentration over days. However, we attempted to minimise this by asking subjects to report to the laboratory in the same time every morning, in which blood collection was in rested state. Third, small sample size could be one of study limitations; however our results showed a good reliability of ICC.

\section{Conclusions}

This study has assessed the within sample, within day and between day reliability of plasma exosomes concentration in healthy male individuals. We showed that exosomes isolated from blood plasma are reliable and can be a good indicator to search for vesicular biomarker. Also, we showed that freeze condition had no effect on size and number of plasma exosome.

\section{Acknowledgments}

I am grateful to my supervisors Professor Helen Dawes and Dr. Ryan Pink whom experience and continuous support helped to accomplish this work. I would like also to thank Dr. Johnny Collett for his continuous support and help in data analysis and study finalising. Also, I would like to thank the Saudi Cultural Bureau in London for their administrative 
and financial support. Finally, I thank all participants in this study for their kind cooperation.

\section{References}

[1] Azmi, A.S., B. Bao, and F.H. Sarkar, Exosomes in cancer development, metastasis, and drug resistance: a comprehensive review. Cancer and Metastasis Reviews, 2013. 32(3-4): p. 623-42.

[2] Théry, C., L. Zitvogel, and S. Amigorena, Exosomes: composition, biogenesis and function. Nature Reviews Immunology, 2002. 2(8): p. 569-79.

[3] Johnstone, R.M., et al., Vesicle formation during reticulocyte maturation. Association of plasma membrane activities with released vesicles (exosomes). Journal of Biological Chemistry, 1987. 262(19): p. 9412-9420.

[4] Sadallah, S., C. Eken, and J. Schifferli, Ectosomes as modulators of inflammation and immunity. Clinical \& Experimental Immunology, 2011. 163(1): p. 26-32.

[5] Lässer, C., M. Eldh, and J. Lötvall, Isolation and characterization of RNA-containing exosomes. JoVE (Journal of Visualized Experiments), 2012(59): p. e3037-e3037.

[6] Logozzi, M., et al., High levels of exosomes expressing CD63 and caveolin-1 in plasma of melanoma patients. PloS one, 2009. 4(4): p. e5219.

[7] Kobayashi, M., et al., In vitro immunological and biological evaluations of the angiogenic potential of platelet-rich fibrin preparations: a standardized comparison with PRP preparations. International Journal of Implant Dentistry, 2015. 1(1): p. 1.

[8] Ohno, S.-i., A. Ishikawa, and M. Kuroda, Roles of exosomes and microvesicles in disease pathogenesis. Advanced drug delivery reviews, 2013. 65(3): p. 398-401.

[9] Kharaziha, P., et al., Tumor cell-derived exosomes: a message in a bottle. Biochimica et Biophysica Acta (BBA)-Reviews on Cancer, 2012. 1826(1): p. 103-111.

[10] Taylor, D.D. and C. Gercel-Taylor, MicroRNA signatures of tumor-derived exosomes as diagnostic biomarkers of ovarian cancer. Gynecologic oncology, 2008. 110(1): p. 13-21.

[11] Ren, Y., et al., Exosomal-like vesicles with immune-modulatory features are present in human plasma and can induce CD4+ T-cell apoptosis in vitro. Transfusion, 2011. 51(5): p. 1002-1011.

[12] Khan, S., et al., Plasma-derived exosomal survivin, a plausible biomarker for early detection of prostate cancer. PloS one, 2012. 7(10): p. e46737.

[13] Raposo, G. and W. Stoorvogel, Extracellular vesicles: exosomes, microvesicles, and friends. The Journal of cell biology, 2013. 200(4): p. 373-383.

[14] Bobrie, A., et al., Exosome secretion: molecular mechanisms and roles in immune responses. Traffic, 2011. 12(12): p. 1659-1668.

[15] Théry, C., Exosomes: secreted vesicles and intercellular communications. F1000 Biol Rep, 2011. 3(15): p. 130.

[16] Stoorvogel, W., Functional transfer of microRNA by exosomes. Blood, 2012. 119(3): p. 646-648.

[17] Ündar, L., et al., Circadian variations in natural coagulation inhibitors protein $C$, protein $S$ and antithrombin in healthy men: a possible association with interleukin-6. Thrombosis and haemostasis, 1999. 81(4): p. 571-575.

[18] Picotte, M., C.G. Campbell, and W.G. Thorland, Day-to-day variation in plasma interleukin-6 concentrations in older adults. Cytokine, 2009. 47(3): p. 162-165.

[19] Gardiner, C., et al., Extracellular vesicle sizing and enumeration by nanoparticle tracking analysis. Journal of extracellular vesicles, 2013. 2.

[20] Bala, S., et al., Circulating microRNAs in exosomes indicate hepatocyte injury and inflammation in alcoholic, drug-induced, and inflammatory liver diseases. Hepatology, 2012. 56(5): p. 1946-1957.

[21] Ploeger, H.E., et al., The effects of acute and chronic exercise on inflammatory markers in children and adults with a chronic inflammatory disease: a systematic review. Exerc Immunol Rev, 2009. 15(1): p. 6-41.

[22] Aoi, W., et al., Muscle-enriched microRNA miR-486 decreases in circulation in response to exercise in young men. Front Physiol, 2013. 4: p. 80.

[23] Théry, C., et al., Isolation and characterization of exosomes from cell culture supernatants and biological fluids. Current protocols in cell biology, 2006: p. 3.22. 1-3.22. 29.

[24] Rani, S., MicroRNA profiling of exosomes isolated from biofluids and conditioned media. RNA Mapping: Methods and Protocols, 2014: p. 131-144.

[25] Shrout, P.E. and J.L. Fleiss, Intraclass correlations: uses in assessing rater reliability. Psychol Bull, 1979. 86(2): p. 420-8.

[26] Hopkins, W.G., Measures of reliability in sports medicine and science. Sports medicine, 2000. 30(1): p. 1-15.

[27] Batterham, A.M. and K.P. George, Reliability in evidence-based clinical practice: a primer for allied health professionals $\approx$. Physical therapy in Sport, 2003. 4(3): p. 122-128.

[28] Kalra, H., et al., Comparative proteomics evaluation of 
plasma exosome isolation techniques and assessment of the stability of exosomes in normal human blood plasma. Proteomics, 2013. 13(22): p. 3354-3364.

[29] Sarker, S., et al., Placenta-derived exosomes continuously increase in maternal circulation over the first trimester of pregnancy. Journal of translational medicine, 2014. 12(1): p. 1 .

[30] Marcos, A., E. Nova, and A. Montero, Changes in the immune system are conditioned by nutrition. European journal of clinical nutrition, 2003. 57: p. S66-S69. 